\title{
Capacité des équipements commerciaux et taux d'exploitation des centres d'approvisionnement intraurbains
}

\section{Introduction}

La présente étude a pour objectif l'examen de la structure d'approvisionnement en biens de consommation d'usage très courant en ville de SaintGall. Dans une première phase de travail, nous avons déterminé ia capacité des équipements commerciaux pour chaque centre d'approvisionnement (sans tenir compte du centre urbain principal*). Dans une phase ultérieure, le taux d'exploitation (théorique) de la capacité commerciale a été déduit moyennant la juxtaposition des capacités en cause avec le nombre d'habitants à l'intérieur des zones d'approvisionnement *) correspondantes.

$\mathrm{Ce}$ travail demeure de caractère monographique, puisqu'il ne porte que sur la ville de Saint-Gall et n'est qu'un diagnostic de la situation actuelle. Il est cependant prévu d'inscrire cette étude dans un cadre plus large qui permettrait d'établir des comparaisons dans l'espace et dans le temps afin de dégager les mécanismes qui régissent l'évolution du système d'approvisionnement urbain.

\section{Méthode de travail}

Parmi les éléments de l'offre susceptibles de déterminer la structure et la capacité des équipements commerciaux d'un centre, citons avec Tietz ${ }^{1)}$ :

a. les propriétés caractérisant la base de l'activité économique (Bestandesmerkmale)

Exemples:

- le nombre de commerces de détail

- les surfaces des commerces de détail resp. les surfaces de vente

- l'effectif du personnel

b. les propriétés rattachées au rendement de l'activité économique (Merkmale der Ausbringung)

*) Afin d'alléger le texte nous reléguons dans un appendice un certain nombre de remarques explicatives complémentaires. Les passages renvoyés dans l'appendice sont marqués d'un astérisque.

1) Cf. Tietz B. Konsument und Einzelhandel, cité d'après Facchinetti B. (1977), p. 25.

2) Cf. Facchinetti B. (1977), p. 79

3) Cf. Facchinetti B (1977), p. 83
Exemples:

- le chiffre d'affaires

- le résultat de l'exploitation.

La capacité d'un service dépend de nombreux facteurs presque impossibles à cerner, tant est grande la variété des structures de ces activités de services. Etant donné l'importance du facteur main-d'œuvre pour la distribution des biens dans le commerce de détail, et qu'en outre beaucoup de services du commerce de détail ne peuvent pas être chiffrés (p. ex. les prestations accessoires comme la livraison à domicile), il n'est pas possible de déterminer la capacité des équipements commerciaux sans avoir recours à des mesures auxiliaires. Ces dernières ne permettent toutefois pas de déterminer la capacité d'une manière exacte, mais elles se prêtent du moins à la caractériser ${ }^{2}$.

Nous nous proposons de concentrer notre attention sur le cas de l'approvisionnement de la population en denrées alimentaires. Dans le cadre de ce travail, la capacité des équipements commerciaux au niveau de la branche alimentation est définie comme le nombre d'habitants qu'un centre peut théoriquement desservir. Comme indicateur de capacité, nous choisissons le nombre de personnes occupées dans les commerces alimentaires et spécialisés. Ceci se justifie d'une part par l'intensité du facteur travail dans la branche faisant l'objet de cette étude et, d'autre part, par l'accessibilité des données de base. Une remarque s'impose toutefois quant aux unités de vente libre-service. Les différences de productivité du personnel occupé dans les diverses formes de distribution impliquent une appréciation trop approximative des capacités commerciales. Afin de pouvoir parer à ce fait dans une certaine mesure, nous avons désagrégé la branche alimentation dans une seconde partie de notre étude.

L'élément "surface de vente», lorsqu'il est considéré isolément, n'est pas un indicateur représentatif de la capacité commerciale, puisque les productivités des surfaces divergent davantage que les productivités de la main-d'œuvre ${ }^{3)}$. Bien que la

Jean-Pierre Jetzer, Forschungsstelle für Wirtschaftsgeographie und Raumplanung an der Hochschule St. Gallen, General-Guisan-Straße 70, 9010 St. Gallen 
surface de vente puisse être considérée comme le complément idéal du facteur main-d'œuvre, nous n'avons pas pu en tenir compte, n'ayant pas d'accès facile aux données de base. Il en va de même pour le chiffre d'affaires et le résultat de l'exploitation.

\section{Application}

\subsection{Centralité du commerce de détail (Einzelhan- delszentralität Z) d'après Tietz}

En nous fondant sur Tietz ${ }^{4)}$ nous déterminons $Z$ pour la branche alimentation dans chaque centre d'approvisionnement:

*) $\mathrm{Z}=\frac{\frac{\begin{array}{c}\text { personnel occupé dans le commerce alimentaire } \\ \text { dans l'espace partiel }\end{array}}{\text { habitants dans l'espace partiel }}}{\frac{\text { personnel occupé dans le commerce alimentaire }}{\text { dans l'espace urbain }}}$

La valeur $\mathrm{Z}$ indique l'excédent ou le déficit d'approvisionnement, mesuré en nombre d'habitants, pour l'espace partiel en question. Admettons un coefficient 2; nous en déduisons que le centre d'approvisionnement dessert théoriquement le double du nombre d'habitants de l'espace partiel correspondant. Un coefficient $>1$ indique une centralité du commerce de détail supérieure à la moyenne et est un indice pour un centre d'approvisionnement dont la zone d'influence dépasse l'espace partiel.

L'analyse de la branche alimentation de manière globale à l'aide du chiffre-indice structurel $\mathrm{Z}$ se fonde sur l'hypothèse de productivités identiques à l'intérieur de la branche en cause, ainsi que sur l'hypothèse partant de l'analogie des structures des besoins dans les zones d'approvisionnement.

Dans le tableau 1 figurent les valeurs de $\mathrm{Z}$ reproduites par centre d'approvisionnement pour la branche alimentation. Sur la base de ces données, il est aisé d'aboutir aux capacités de la branche examinée et aux taux d'exploitation (te) respectifs. Les chiffres du tableau mentionné ci-dessus renseignent sur ces valeurs dérivées.

Tableau 1: Résumé des valeurs de la capacité commerciale et du taux d'exploitation en fonction des centres d'approvisionnement

\begin{tabular}{|l|l|l|l|l|l|l|l|l|l|l|}
\hline classification & rc 4 & rc 3 & & & & & rc 2 & & & \\
\hline $\begin{array}{l}\text { centre } \\
\text { valeurs }\end{array}$ & $\begin{array}{l}\text { Gross- } \\
\text { acker/ } \\
\text { St. Fiden } \\
\text { S }{ }^{*} \text { ) }\end{array}$ & $\begin{array}{l}\text { Singen- } \\
\text { berg }\end{array}$ & Lachen & $\begin{array}{l}\text { Lang- } \\
\text { gasse }\end{array}$ & Neudorf & $\begin{array}{l}\text { St. Geor- } \\
\text { gen }\end{array}$ & $\begin{array}{l}\text { St. Oth- } \\
\text { mar/ } \\
\text { Ober- } \\
\text { str. }\end{array}$ & $\begin{array}{l}\text { Heilig- } \\
\text { kreuz }\end{array}$ & $\begin{array}{l}\text { Bruggen } \\
\text { S }\end{array}$ & Krontal \\
\hline za & 6200 & 3400 & 3400 & 3300 & 2600 & 2400 & 3000 & 2400 & 1900 & 1700 \\
\hline méthode Tietz & & & & & & & & & & \\
$Z$ & 0.963 & 0.385 & 1.523 & 0.514 & 1.018 & 0.991 & 0.486 & 0.193 & 1.303 & 0.606 \\
C & 6000 & 1300 & 5200 & 1700 & 2700 & 2400 & 1500 & 500 & 2500 & 1000 \\
te & 1.04 & 2.60 & 0.66 & 1.95 & 0.98 & 1.01 & 2.06 & 5.18 & 0.77 & 1.65 \\
\hline $\begin{array}{l}\text { méthode dés- } \\
\text { agrégée }\end{array}$ & & & & & & & & & & \\
$\begin{array}{l}\text { C } \\
\text { te }\end{array}$ & 5300 & 1300 & 5200 & 3000 & 2400 & 2600 & 2200 & 1500 & 3000 & 2200 \\
& 1.17 & 2.61 & 0.66 & 1.11 & 1.09 & 0.93 & 1.36 & 1.66 & 0.64 & 0.76 \\
\hline
\end{tabular}

\section{Légende:}

$\mathrm{rc}=$ rang des centres d'approvisionnement

za = habitants à l'intérieur de la zone d'approvisionnement

$\mathbf{Z}$ = centralité du commerce de détail d'après Tietz

C = capacité commerciale du centre d'approvisionnement

te $=$ taux d'exploitation de la capacité commerciale
4) Cf. Tietz B. (1974), col. 1907 sqq.

\section{*) Grossacker/St. Fiden S}

Les centres d'approvisionnement Grossacker (centre commercial intégré) et St. Fiden $\mathrm{S}$ ont été résumés en un centre bipolaire dans le cadre de l'analyse de la capacité commerciale étant donné qu'il y a superposition des aires d'approvisionnement.

\section{Annotation}

Comme l'on pouvait s'y attendre, la variation des valeurs entre les deux modes de calcul utilisés réagit à raison inverse avec le degré d'équipement des centres. Ce n'est donc que sur la base d'une approche différenciée, apte à laisser transparaître la structure de l'équipement des centres intraurbains, que des valeurs appropriées pourront être forunies. 
Tableau 1: Résumé des valeurs de la capacité commerciale et du taux d'exploitation en fonction des centres d'approvisionnement (suite)

\begin{tabular}{|c|c|c|c|c|c|c|c|c|c|}
\hline classification & rc 2 (sui & & & & re 1 & & & & \\
\hline valeurs centre & \begin{tabular}{|l|}
$\begin{array}{l}\text { Rot- } \\
\text { monten }\end{array}$ \\
\end{tabular} & \begin{tabular}{|l|} 
St. Fiden \\
$\mathrm{N}$
\end{tabular} & Winkeln & \begin{tabular}{|l} 
Riet- \\
hüsli
\end{tabular} & Achslen & Zil & Schoren & \begin{tabular}{|l} 
Hinter- \\
berg
\end{tabular} & $\begin{array}{l}\text { Brruggen } \\
\mathbf{N}\end{array}$ \\
\hline $\mathrm{za}$ & 1700 & 1300 & 1100 & 1100 & 1200 & 1200 & 1600 & 1200 & 300 \\
\hline $\begin{array}{l}\text { méthode Tietz } \\
\text { Z } \\
\text { C } \\
\text { te }\end{array}$ & $\begin{array}{l}0.606 \\
1000 \\
1.65\end{array}$ & $\begin{array}{l}0.725 \\
1000 \\
1.38\end{array}$ & $\begin{array}{l}1.064^{\circ} \\
1200 \\
0.94\end{array}$ & $\begin{array}{l}1.046 \\
1100 \\
0.96\end{array}$ & $\begin{array}{l}0.303 \\
400 \\
3.30 \\
\end{array}$ & $\begin{array}{l}0.229 \\
300 \\
4.37 \\
\end{array}$ & $\begin{array}{l}0.275 \\
500 \\
3.64 \\
\end{array}$ & \begin{tabular}{|l}
0.156 \\
200 \\
6.41 \\
\end{tabular} & \begin{tabular}{|l|}
0.844 \\
300 \\
1.18 \\
\end{tabular} \\
\hline $\begin{array}{l}\text { méthode dés- } \\
\text { agrégée } \\
\text { C } \\
\text { te }\end{array}$ & $\begin{array}{l}1500 \\
1.12\end{array}$ & $\begin{array}{l}2900 \\
0.43\end{array}$ & \begin{tabular}{|l|}
1700 \\
0.68
\end{tabular} & $\begin{array}{l}1600 \\
0.64\end{array}$ & $\begin{array}{l}1200 \\
1.02\end{array}$ & $\begin{array}{l}900 \\
1.39\end{array}$ & $\begin{array}{l}900 \\
1.93\end{array}$ & $\begin{array}{l}600 \\
2.07 \\
\end{array}$ & $\begin{array}{l}900 \\
0.37\end{array}$ \\
\hline
\end{tabular}

Remarques générales:

- si $Z>1 \longrightarrow$ te $<1$, c'est-à-dire que l'équipement commercial du centre tend vers une surcapacité par rapport à sa zone d'approvisionnement et que l'exploitation de la capacité dépend de la demande extérieure

- si $\mathrm{Z}=1 \longrightarrow$ te $=1$, c'est-à-dire qu'il y a équilibre entre l'offre et la demande de capacités à l'intérieur de la zone d'approvisionnement

- si Z $<1 \rightarrow$ te $>1$, c'est-à-dire que le centre d'approvisionnement est sous-équipé par rapport à la zone à desservir.

La valeur $\mathrm{Z}$ met en évidence le degré de dotation des centres d'approvisionnement en équipements commerciaux de la branche alimentation à un moment donné par rapport aux zones à desservir. Il est incontestable que ce genre de chiffre-indice n'a qu'une valeur descriptive, mais nullement explicative. Malgré cette restriction nous nous servons de cet indice structurel en tant que première approche, afin de donner un aperçu de l'organisation spatiale des services sur le plan de l'approvisionnement en biens alimentaires.

\subsection{Détermination de la capacité des équipements commerciaux et du taux d'exploitation par diffé- renciation des données de base}

En vue de fonder nos réflexions sur une base différenciée, nous subdivisons la branche alimentation en sous-branches (en partie selon des critères institutionnels [Forme de distribution], en partie selon des critères fonctionnels [nature des biens]):

- commerces d'alimentation générale ${ }^{* *}$ )

- grands distributeurs du commerce alimentaire $* *$ )

- boulangeries/pâtisseries

- boucheries/charcuteries.
Au moyen de la désagrégation de la branche alimentation, il est possible, dans une certaine mesure, de tenir compte des différences de productivité de la main-d'œuvre occupée dans les diverses formes de distribution. En outre, la différenciation permet de mieux refléter la structure du commerce de détail dans les centres d'approvisionnement.

Par la suite, nous définissons la productivité du personnel comme le nombre d'habitants desservis en moyenne par un employé de vente occupé dans une sous-branche alimentation. Nous déterminons d'abord la producitivité (p) d'un employé de vente, (e) pour chaque sous-branche alimentation (i):

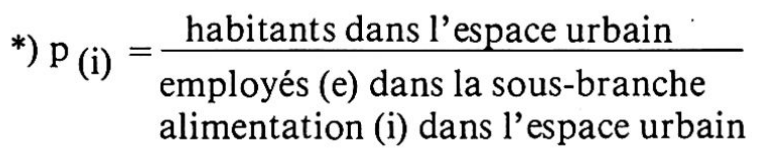

La capacité moyenne (C) d'un centre d'approvisionnement résulte de l'addition des productivités (p) selon le nombre d'employés (e) dans les sousbranches alimentation (i) et en divisant le total ainsi obtenu par le nombre (n) des sous-branches représentées dans le centre d'approvisionnement examiné:

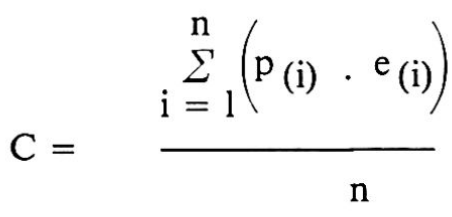

**) Nous nous référons à la structure usuelle de l'organisation distributrice du commerce de détail:

a. le petit et moyen commerce de détail: commerces de détail indépendants, soit organisés en chaînes volontaires ou en groupements d'achats, soit nonorganisés

b. les grands distributeurs de l'alimentation: organisations disposant d'un vaste réseau de filiales et contrôlant une importante part du marché.

Source: Centonze E. (1978), p. 166 sq. 
Le taux d'exploitation (te) de la capacité commerciale (C) d'un centre d'approvisionnement s'obtient par la juxtaposition de la capacité en cause avec le nombre d'habitants de la zone d'approvisionnement (za) correspondante:

habitants za

te $=$ C

Le taux d'exploitation indique dans quelle mesure la capacité commerciale d'un centre d'approvisionnement est exploitée resp. épuisée par la demande déployée à l'intérieur de la zone à desservir. Cette construction part du postulat suivant: la couverture de la demande de biens d'utilisation courante et générale doit être possible dans un rayon accessible aux piétons.

La mobilité spatiale en particulier, et son influence sur le comportement des consommateurs, apportent des modifications considérables dans ce modèle d'approvisionnement. Ce n'est que dans le cadre d'une analyse plus étendue, englobant d'une part l'observation de l'ampleur et de l'orientation spatiale des flux de consommateurs et, d'autre part, la demande au niveau périodique et apériodique, que l'approche de la réalité complexe pourrait s'effectuer.

\section{Présentation des résultats}

\subsection{Interprétation générale}

Dans le tableau 1 nous avons résumé les données relatives à l'équipement des centres d'approvisionnement. Nous nous restreignons à l'interprétation des trois cas-types suivants:

- 1er cas: centres d'approvisionnement dont le te $>1$ indique un sous-équipement relatif

- 2e cas: centres d'approvisionnement dont le te $=1$ indique un équilibre des capacités

- 3e cas: centres d'approvisionnement dont le te $<1$ indique une surcapacité relative.

ler cas: te $>1$

Comme nous l'avons constaté antérieurement, un taux d'exploitation supérieur à 1 indique un souséquipement relatif du centre d'approvisionnement. Cette situation peut être imputée aux faits suivants (l'énumération n'étant pas exhaustive):

- l'attraction d'un centre voisin concurrentiel absorbe une partie du pouvoir d'achat de la zone d'approvisionnement attribuée au centre examiné

- le seuil critique, c'est-à-dire le besoin d'un réservoir minimal de clientèle (densité de la population résidente et du pouvoir d'achat) pour assu- rer un fonctionnement correct d'un service central, n'est pas atteint dans la zone d'approvisionnement environnante.

Afin de déceler les relations existant entre l'équipement d'un centre d'approvisionnement et son taux d'exploitation, il s'avère nécessaire de tenir compte des exigences des consommateurs. Il est à retenir que ces exigences, qui se traduisent par les habitudes d'achat, varient tant dans l'espace (c'est-àdire suivant la structure socio-démographique des unités spatiales) que dans le temps, et que, justement, la variation et le dynamisme sont les forcesclés qui affectent les relations concurrentielles à l'intérieur du système des centres d'approvisionnement. Par analogie avec la théorie des «Zentrale Orte» (W. CHRISTALLER) ${ }^{5)}$, les centres intraurbains ne sont pas de qualité identique, mais forment une hiérarchie à plusieurs degrés dont les zones d'influence se chevauchent. Il ne peut dès lors être admis que les usagers potentiels s'orientent nécessairement vers le centre le plus proche. Il nous semble indiqué d'admettre une certaine indétermination dans le comportement des acteurs. Pour tenir compte de cette indétermination, on a recours à une approche probabilistique où le comportement spatial est fonction de l'attraction relative des centres et de la distance entre la localisation de l'usager potentiel et des centres à sa portée, c'est-à-dire l'accessibilité des centres ${ }^{\text {O }}$.

\section{2e cas: te $=1$}

C'est le cas marquant l'adaptation de la capacité des équipements de la branche alimentation à la demande déployée à l'intérieur de la zone d'approvisionnement correspondante. Cette situation permet de conclure à une certaine autonomie du centre dans l'approvisionnement de la population à l'échelon de la gamme des services courants.

$\mathrm{Ce}$ cas limite pourrait se produire, au niveau de l'espace urbain, en supposant un réseau d'approvisionnement cohérent dont chaque centre serait pourvu du même pouvoir d'attraction et n'assurerait la couverture de la consommation journalière que pour sa propre zone d'approvisionnement.

Une autre situation, de caractère hypothétique, serait le cas de flux de pouvoir d'achat compensatoires.

\section{3e cas: te $<1$}

Un taux d'exploitation inférieur à 1 laisse apparaître une surcapacité relative du centre d'approvisionnement, c'est-à-dire que la portée des services centraux dépasse la zone d'approvisionnement immédiate.

\footnotetext{
5) Cf. Christaller W. (1933).

6) Cf. Bunge W. (1966), p. 143 et BMBau (1974), p. 64 sqq.
} 
Il faut préciser à cet égard que l'interprétation du taux d'exploitation des équipements centraux varie suivant le rang du centre en cause. Ainsi, pour un centre de rang élevé, il est significatif que la zone d'influence embrasse une plus grande portion de l'espace que la zone d'approvisionnement proprement dite. Au contraire, pour un centre de rang inférieur, une surcapacité relative ne peut guère être imputée à un excédent d'importance du centre dont le rayonnement dépassant la zone d'approvisionnement immédiate serait basé sur des relations fondamentales ${ }^{7)}$.

La situation réelle est sans doute le mieux reflétée par les cas indiquant une disparité entre l'offre et la demande de capacités en équipements centraux par rapport aux zones d'approvisionnement immédiates. Ceci en raison même du fait que le réseau d'approvisionnement urbain s'est organiquement inséré dans le tissu du réseau d'habitat, perturbant, par conséquent, le schéma hexagonal tracé par w. CHRISTALlER. D'autre part, les, zones d'approvisionnement ont été définies pour la branche alimentation au moyen de critères normatifs, ce qui explique en partie le décalage de l'équipement commercial.

\subsection{Exploitation des résultats}

Ci-après nous avons recours à la division ternaire appliquée auparavant pour grouper les centres d'approvisionnement d'après le taux d'exploitation de leurs équipements centraux de la branche alimentation. Afin d'étendre nos considérations au problème de la qualité de l'approvisionnement, nous procédons au découpage supplémentaire par rapport au rang de centres.

Il convient, à ce propos, de soulever la question de savoir si la dotation des centres en équipements de la branche alimentation est suffisante pour répondre aux besoins de la population résidente dans les zones d'approvisionnement au niveau qualitatif correspondant. Sur la base des données du tableau 2, on peut affirmer que sur les 19 centres d'approvisionnement faisant l'objet de notre étude, 9 se distinguent par un sous-équipement relatif. A eux seuls, les degrés 4 et 3 de la classification des centres réunissent la moitié, c'est-à-dire 3 centres de cette catégorie assurant une qualité d'approvisionnement optimale. La situation se révèle particulièrement précaire dans les quartiers résidentiels nouvellement érigés (par exemple les quartiers de Zil, Hinterberg) où l'approvisionnement en denrées alimentaires est assuré par des points de vente isolés qui ne sont pas en mesure d'offrir un choix relativement complet de services utilisés quotidiennement, afin de procurer aux habitants un approvisionnement de qualité minimale.
Tableau 2:

typologie des centres d'approvisionnement

\begin{tabular}{|c|c|l|l|}
\hline re & $>1$ & $\sim 1$ & $<1$ \\
\hline 4 & $\begin{array}{c}41 \text { Grossacker/ } \\
\text { St. Fiden S }\end{array}$ & & \\
\hline 3 & $\begin{array}{l}31 \text { Singenberg } \\
33 \text { Langgasse }\end{array}$ & $\begin{array}{l}34 \text { Neudorf } \\
35 \text { St. Georgen }\end{array}$ & 32 Lachen \\
\hline 2 & $\begin{array}{l}21 \text { St. Othmar/ } \\
\text { Oberstrasse } \\
22 \text { Heiligkreuz } \\
25 \text { Rotmonten }\end{array}$ & $\begin{array}{l}23 \text { Bruggen S } \\
24 \text { Krontal } \\
26 \text { St. Fiden N } \\
27 \text { Winkeln } \\
28 \text { Riethüsli }\end{array}$ \\
\hline 1 & $\begin{array}{l}12 \text { Zil } \\
13 \text { Schoren } \\
14 \text { Hinterberg }\end{array}$ & 11 Achslen & 15 Bruggen N \\
\hline
\end{tabular}

$\mathrm{rc}=$ rang des centres d'approvisionnement

te $=$ taux d'exploitation de la capacité commerciale

Cf. pour la classification des centres Boesch M. (1980) et Bugmann E. (1980).

Lachen est le seul centre d'approvisionnement parmi les rangs 4 et 3 qui appartienne à la catégorie des centres caractérisés par une surcapacité relative. Sa zone d'influence s'étend ainsi tendantiellement au-delà de la zone d'approvisionnement immédiate. Il est en outre significatif que les services centraux de la branche alimentation du centre bipolaire Grossacker/St. Fiden S, malgré un équipement quasiment complet au niveau des besoins périodiques et en partie apériodiques (surtout en ce qui concerne le centre commercial intégré Grossacker), ne déployent pas de rayonnement dépassant la zone d'approvisionnement proprement dite.

Au $2 e$ échelon de la classification des centres d'approvisionnement, les localisations de Bruggen S, Krontal, St.Fiden N, Winkeln et Riethüsli accusent une surcapacité relative. Les zones d'approvisionnement respectives ne sont que peu densément habitées, ce qui exige de la part des services centraux une aire d'influence plus vaste afin de pouvoir assurer la clientèle nécessaire à leur rentabilité ou à leur plein fonctionnement. Contrairement aux centres de rang supérieur, il ne peut être question d'une centralité élevée comme une analyse isolée de la branche alimentation pourrait le faire apparaître. L'équipement des centres de rang inférieur ne profite pas de l'effet d'entraînement exercé par l'agglomération de services de niveau

7) $\mathrm{Cf}$. pour les catégories de relations entre la ville et la région Beaujeu-Garnier J./Chabot G. (1963), p. 442 sq. 
supérieur dont l'implantation confère au centre un pouvoir d'attraction rayonnant au-delà de la zone d'approvisionnement immédiate. D'où nous déduisons la nécessité de considérer l'équipement des centres comme un tout particulièrement complexe et non comme une addition hétéroclite d'éléments sans rapport les uns avec les autres.

Les seuls centres d'approvisionnement qui, du moins dans le cadre de la présente étude, disposent d'un équipement de la branche alimentation relativement adapté aux besoins quantifiables de la population à l'intérieur de la zone à desservir, et assurant un approvisionnement de base à un niveau qualitatif suffisant, sont les centres de St. Georgen et Neudorf.

Comme on l'a déjà relevé, notre interprétation recommande, de façon manifeste, une étude complémentaire englobant l'analyse des caractéristiques locales, tant du point de vue purement spatial (par exemple localisation du centre examiné dans l'aire d'influence d'un centre de rang supérieur, effectif démographique) qu'au point de vue socio-économique (structure des âges, catégories socio-professionnelles, structure des revenus de la population résidente à l'intérieur de la zone d'approvisionnement).

Au vu des données du tableau 1, il existe des divergences considérables quant à l'exploitation des équipements commerciaux. Il est intéressant d'examiner ces divergences en fonction du rang des centres d'approvisionnement.

Taux d'exploitation (te) par rapport au rang des centres:

- te au niveau du 4e rang: 1.17

- te au niveau du $3 \mathrm{e}$ rang: 1.05

- te au niveau du 2e rang: 0.85

( - te au niveau du ler rang: 1.28 ).

Abstraction faite du cas spécial des centres du ler degré (quartiers résidentiels relativement nouveaux où les frais de démarrage pour l'équipement des centres sont élevés par le fait même que la localisation n'est souvent pas combinée à la présence d'une clientèle de base déjà nombreuse, ce qui explique leur sous-équipement relatif ${ }^{8)}$, nous constatons une relation inverse entre la capacité de l'équipement commercial et le rang des centres. En d'autres termes, plus le rang des centres est bás, plus leur tendance vers une surcapacité sera prononcée. En outre, il faut souligner le lien étroit qui existe entre la densité de la population à l'intérieur de la zone d'approvisionnement et la capacité commerciale du centre correspondant. Ainsi, dans les zones d'approvisionnement peu densément habitées, les centres tendent en général vers une surcapacité relative, ce qui semble confirmer la règle selon laquelle il existe une corrélation positive entre le rang des centres et l'effectif démographique à l'intérieur de l'aire d'influence.
La carte ainsi que le tableau 3 ci-après résument la situation concernant l'insertion du réseau des centres d'approvisionnement dans le contexte urbain. Il convient de relever à ce propos le "vide» en matière d'approvisionnement au niveau qualitatif existant dans la partie Ouest de la ville de Saint-Gall.

Tableau 3: répartition des centres d'approvisionnement dans l'espace

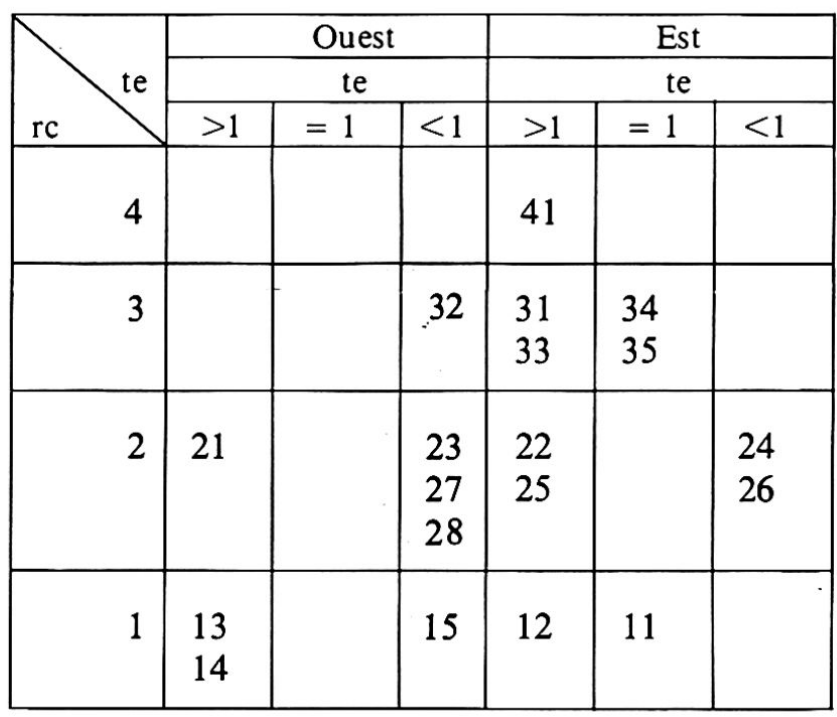

$\mathrm{rc}=$ rang des centres d'approvisionnement

te $=$ taux d'exploitation de la capacité commerciale Numérotage des centres: cf. tableau 2

\section{Remarques finales}

Cette partie de l'étude a eu pour objet la détermination de la capacité de l'équipement commercial de la branche alimentation dans les centres d'approvisionnement en ville de Saint-Gall (sans tenir compte du centre urbain principal). En tant que complément de la première partie, traitant la problématique de l'approvisionnement avant tout dans la perspective de la demande (accessibilité des centres, qualité d'approvisionnement; cf. Boesch M. 1980), nous nous sommes efforcés d'éclaircir l'objet de notre étude sous l'aspect de l'offre. La détermination des capacités de la branche alimentation pour les centres de notre analyse a été effectuée sur la base d'une approche mise au point par Tietz, approche visant à déterminer la centralité des centres d'approvisionnement au niveau du commerce de détail (Einzelhandelszentralität). En vue de tenir compte, du moins en partie, de la variation de la structure de l'équipement des centres, nous avons quelque peu adapté l'approche. A partir de la centralité ainsi déterminée, il était aisé de dériver le taux d'exploitation (théorique) de l'équi-

8) Cf. Arbeitskreis Gewerbliche Ansiedlung Köln (1977). 


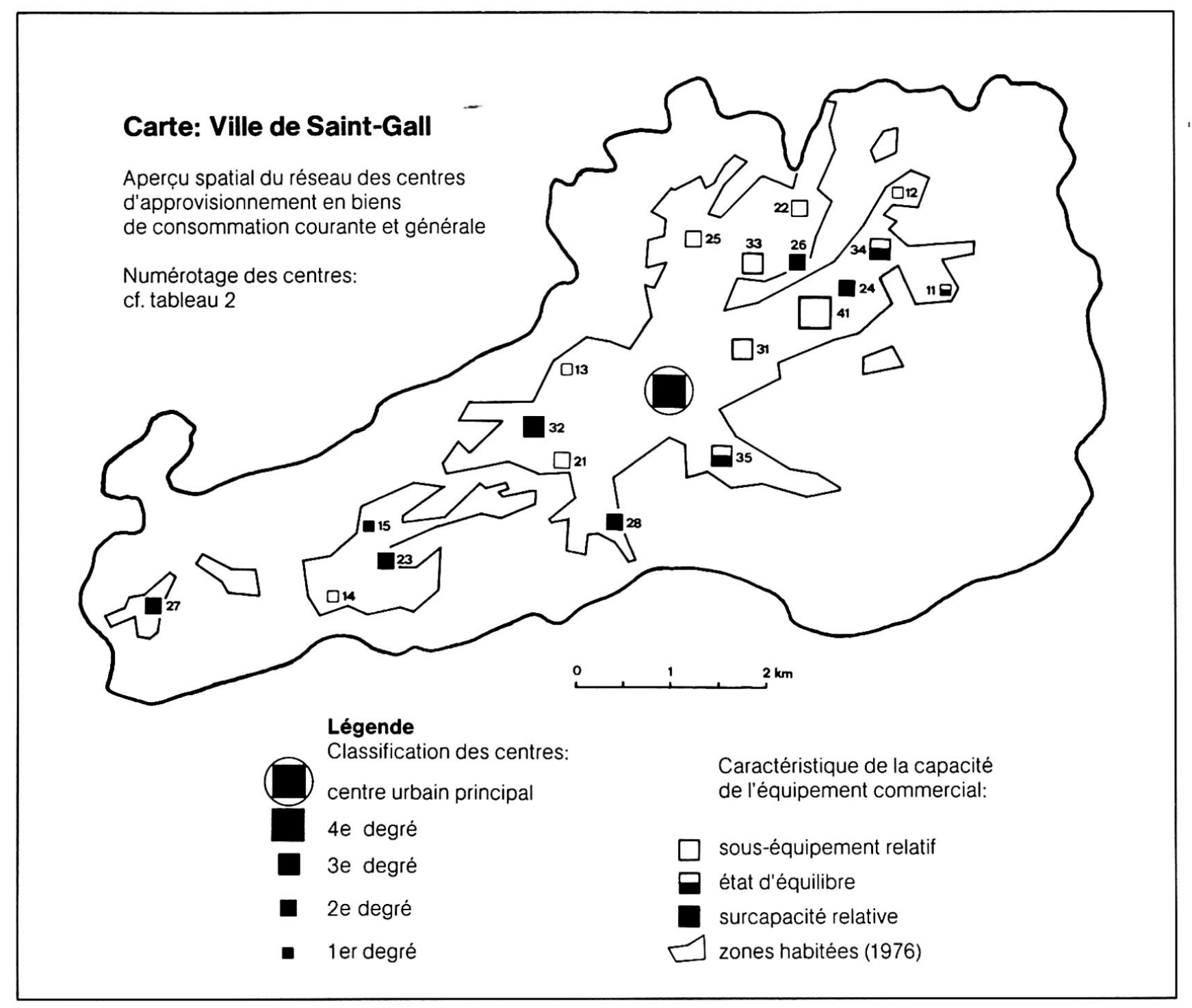

pement commercial de la branche alimentation des centres étudiés.

Les résultats de notre étude ont tous un caractère hypothétique. Aussi bien les données de base que l'approche utilisée ne permettent pas de tirer des conclusions décisives quant à l'adaptation du réseau des centres d'approvisionnement aux exigences de la demande. Du point de vue du consommateur, il est indispensable d'étendre l'horizon de l'analyse au-delà de la couverture des besoins quotidiens. La concentration spatiale de services centraux aptes à faire face à la demande à tous les degrés de périodicité implique un effet synergétique qui amplifie l'attraction d'un centre. Signalons à ce propos l'importance des «multiple purpose trips» (démarches à fins multiples) ${ }^{9}$. En pratique nous constatons une combinaison des démarches afin d'obtenir, au prix d'un seul déplacement, un éventail de biens complet. Il s'ensuit que l'analyse partielle par catégorie de besoins n'a qu'une valeur purement théorique et n'a d'utilité qu'en tant

9) Cf. Bunge W. (1966), p. 143 et p. 162 sq. que point de départ pour une étude plus approfondie du phénomène d'approvisionnement.

Sans prétendre être exhaustif, nous évoquons sommairement quelques facteurs susceptibles de déterminer le comportement spatial des consommateurs:

- l'auto-attraction du centre d'approvisionnement, celle-ci étant d'autant plus importante qu'il y aura un effet cumulateur sur la base de la densité spatiale des équipements complémentaires

- l'attractivité des centres concurrentiels voisins (il y a lieu de tenir compte du centre urbain principal qui, de par son accessibilité et son attractivité pour le choix qu'il offre entre une grande variété d'activités et de services complémentaires, entraîne une pression excessive exercée par les habitants de toute la région)

- la structure socio-économique à l'intérieur des zones d'approvisionnement.

«Das Aufzeigen von Nachfragestrukturen im Bereich der Grundfunktionen kann nicht losgelöst gesehen werden von den unterschiedlichen Bedürf- 
nissen und Ansprüchen, die sich aus spezifischen demographischen Lagen und sozialen Positionen ergeben.> ${ }^{10}$ (Les structures de la demande relevant $\mathrm{du}$ domaine des fonctions fondamentales ne peuvent être démontrées sans les associer aux différents besoins et prétentions qui résultent des circonstances spécifiques du cadre socio-démographique. Traduction par l'auteur.) Ce n'est que par l'application du procédé empirique d'investigation sociale que les interdépendances à l'intérieur des unités socio-spatiales pourront être saisies, afin d'expliquer le système d'approvisionnement actuel et d'anticiper son évolution.

S'il s'agit en outre de porter un jugement sur le fonctionnement et la viabilité des centres d'approvisionnement, il s'avère nécessaire de déceler les exigences de localisation des exploitants. Dans cette optique, c'est avant tout l'établissement de seuils dans le rapport entre services et clientèle de base (minimale) nécessaire au plein fonctionnement de ces services et à leur rentabilité qui est d'un intérêt primordial. Comparativement aux valeurs indicatives de l'Institut ORL ${ }^{11)}$ les chiffres de notre analyse sont très bas étant donné que les normes ORL représentent des seuils minima et sont en partie dépassées par l'évolution des formes de distribution dans le commerce de détail (cf. nos valeurs dans l'appendice). Ces quelques remarques mettent en évidence que l'appréciation du réseau d'approvisionnement, tant sur le plan de la couverture des besoins qu'au point de vue de l'insertion spatiale, n'est possible qu'en tenant compte des objectifs des deux groupes d'acteurs économiques, utilisateurs et exploitants. Avant de préconiser des formes de distribution plus proches des consommateurs, il s'agit d'élucider les questions relatives à la réalisation de ce postulat, c'est-à-dire la «faisabilité» du point de vue économique, ainsi que l'aspect de la compatibilité avec les habitudes d'achat des usagers.

\section{Appendice}

\section{Remarques explicatives complémentaires relatives} à la base formelle de ce travail

Dans la présente étude, nous nous préoccupons de la situation de l'approvisionnement en biens de consommation d'usage très fréquent au niveau des quartiers en ville de Saint-Gall. Pour être complète notre étude devrait inclure l'analyse de l'influence du centre urbain principal. D'une part, le centre principal exerce un pouvoir d'attraction sur un espace de dimension régionale et, d'autre part, les problèmes sur le plan de l'approvisionnement en

10) Poschwatta W. (1978), p. 199.

11) Cf. Maurhofer F./Leibundgut H. (1969) et Vademecum Institut ORL 1973/74. biens de consommation courante et générale se manifestent particulièrement au niveau des quartiers. $\mathrm{Si}$, pour ces raisons, l'on fait abstraction du centre principal, il convient d'opérer avec les valeurs suivantes:

a. pour l'espace urbain

- le personnel occupé dans la branche alimentation selon l'enquête menée par notre institut

- la population résidente à l'intérieur des zones d'approvisionnement

b. pour l'espace partiel

- le personnel occupé dans la branche alimentation au centre d'approvisionnement délimité pour chaque zone d'approvisionnement

- la population résidente à intérieur de la zone d'approvisionnement respective.

Nous partons des postulats suivants:

- les zones d'approvisionnement sont définies en fonction des exigences des utilisateurs quant à l'accessibilité des centres d'approvisionnement (cf. Boesch M. 1980)

- les flux de pouvoir d'achat entre centres de rang différent sont négligeables au niveau de l'approvisionnement en biens de consommation courante, étant donné que la disposition des consommateurs à faire de longs parcours diminue avec l'augmentation de la fréquence de consommation ${ }^{12)}$

- les exploitants adaptent leurs capacités au potentiel du marché à l'intérieur des zones d'approvisionnement.

Comparaison des données empiriques d'après l'enquête FWR avec les valeurs indicatives de l'Institut $O R L$

\begin{tabular}{lllll}
\hline $\mathrm{p}_{(\mathrm{i})}$ & & $\overline{\mathrm{x}}$ & $\mathrm{c}(\mathrm{FWR})$ & ORL \\
\hline $\mathrm{p}_{(1)}$ & 468 & 2.32 & 1086 & 1000 \\
$\mathrm{p}_{(2)}$ & 292 & 7.83 & 2286 & 5000 \\
$\mathrm{p}_{(3)}$ & 346 & 3.22 & 1114 & 1200 \\
$\mathrm{p}_{(4)}$ & 416 & 3.81 & 1585 & 1500 \\
\hline
\end{tabular}

$p_{(i)}=$ productivité du personnel en fonction des sous-branches alimentation 1 à 4.

1: commerces d'alimentation générale

2: grands distributeurs du commerce alimentaire

3: boulangeries/pâtisseries

4: boucheries/charcuteries

$\overline{\mathrm{x}}=$ moyenne des personnes occupées par point de vente en fonction des sous-branches alimentation

c = capacité moyenne d'un point de vente par sous-branche alimentation

Sources: - enquête FWR (1975/76 et 1977)

- Maurhofer F./Leibundgut H. (1969)

- Vademecum Institut ORL (1973/74) 


\section{Zusammenfassung}

Ziel der Untersuchung ist die Ermittlung der Einzelhandelskapazitäten des Lebensmittelsektors in den dem Hauptgeschäftszentrum von St. Gallen nachgelagerten Subzentren. Die Erfassung der Versorgungssituation von der Angebotsseite her erfolgt auf der Grundlage einer Strukturkennziffer. In einem zweiten Schritt wird der Auslastungsgrad der verfügbaren Kapazitäten im LebensmittelEinzelhandel abgeleitet. Als Datenbasis dient einerseits die Beschäftigtenzahl in den untersuchten Einzelhandelseinrichtungen und andererseits die Bevölkerungszahl innerhalb der normativ abgegrenzten Versorgungsbereiche. Die Konstruktion gründet auf dem versorgungspolitischen Postulat, mindestens den täglichen Bedarf in Fußgängerdistanz decken zu können. Im Rahmen dieser Arbeit, welche die Untersuchung der untersten Stufe der Bedarfsdeckung zum Gegenstand hat, sind die Kaufkraftwanderungen unberücksichtigt geblieben. Die Ergebnisse der vorliegenden Untersuchung, die als Teilstudie einer weiterführenden Arbeit verstanden sein will, haben höchstens tendenzielle Gültigkeit. Sowohl aufgrund der empirischen Basis als auch des methodischen Ansatzes sind keine schlüssigen Aussagen bezüglich der Angemessenheit des räumlichen Versorgungsnetzes möglich. Als erste Annäherung gewinnen die Resultate jedoch insofern praktische Relevanz, als sich am Beispiel der Stadt St. Gallen zeigen läßt, daß die ermittelten Kapazitätsverhältnisse den politisch-normativen Postulaten vielfach nicht bzw. knapp zu entsprechen vermögen.

Gilt es überdies die Funktions- und Lebensfähigkeit von Zentren zu beurteilen, so ist einerseits der Betrachtungshorizont über die rein tägliche $\mathrm{Be}$ darfsdeckung hinaus zu erweitern und andererseits den Ansprüchen sowohl der Zentrumsbenützer als auch der Einrichtungsträger vermehrt Rechnung zu tragen. Erst der Grad der Übereinstimmung der Anforderungsprofile beider Gruppen von Akteuren gibt Aufschluß über den Erfüllungsgrad einer bedürfnis- und siedlungsgerechten Versorgung der Bevölkerung mittels Zentren. Diese Überlegungen zeigen, daß nur ein umfassender Ansatz Transparenz in das städtische Versorgungsgefüge bringen kann.

\section{Bibliographie}

ARBEITSKREIS GEWERBLICHE ANSIEDLUNG KÖLN (ED.) Sicherstellung der Nahversorgung. Die Dekkung des täglichen Bedarfs als Planungsaufgabe und wirtschaftliches Problem. (= Studie 3) Köln 1977.
BEAUJEU-GARNIER J./CHABOTG. Traité de Géographie Urbaine. Paris 1963.

BMBau (ED.) Funktionelle Erfordernisse zentraler Einrichtungen als Bestimmungsgröße von Siedlungs- und Stadteinheiten in Abhängigkeit von Größenordnung und Zuordnung. (= Städtebauliche Forschung 03.003.) Bonn 1972.

BMBau (ED.) Standortwahl und Flächenbedarf des tertiären Sektors in der Stadtmitte. (= Städtebauliche Forschung 03.024.) Bonn 1974.

BOESCH,M. Versorgungsbereiche, Versorgungsqualität und Versorgungsgrad am Beispiel der Einzelhandelsversorgung in der Stadt St. Gallen. In: Geographica Helvetica 35, 2, 1980.

BUGMANN,E. Zur Problematik der intraurbanen Subzentrenforschung. In: Geographica Helvetica $35,2,1980$.

BUNGE, w. Theoretical Geography. (= Lund Studies in Geography, Ser. C., No. 1) Lund 1966.

CENTONZE, E. Die Versorgungsstruktur des Einzelhandels in regionaler Hinsicht. Diss. HSG. Bern 1978.

CHRISTALleR,w. Die Zentralen Orte in Süddeutschland. Jena 1933.

FACCHINETTI, B. Die Ermittlung regionaler Unterschiede in der Versorgung mit Dienstleistungen des Einzelhandels in der Schweiz: Methodologische Probleme der Erfassung. Diss. HSG. St. Gallen 1977.

MAURHOFER, F./LEIBUNDGUT, $\mathrm{H}$. Grundlagen zur Berechnung der Basisbevölkerung zentraler Einrichtungen. ( = Arbeitsberichte zur ORL-Planung, Nr. 7, ORL-ETHZ) Zürich 1969.

POSCHWATTA, W. Verhaltensorientierte Wohnumfelder. In: Geographische Rundschau 30, Heft 5, 1978.

TIETZ, B. Standort. In: Handwörterbuch der Absatzwirtschaft. Tietz B. (Ed.). Stuttgart 1974.

\section{Données de base}

Enquête FWR (= Forschungsstelle für Wirtschaftsgeographie und Raumplanung an der Hochschule St. Gallen)

Institut pour l'aménagement national, régional et local de l'EPFZ, Zurich.

Les données qui servent de base à notre étude ont été recueillies par voie d'enquête aux semestres d'été 1975/76 (enquêtes d'étudiants largement complétées par des recherches personnelles en 1977).

Vademecum. Aménagement du Territoire 1973/74 